\title{
An unprecedented large mesonephric cyst posing diagnostic dilemma and surgical challenge
}

\author{
Kameshwarachari Pushpalatha ${ }^{1}$, Pritinanda Mishra ${ }^{2}$, Nerbadyswari Deep Bag $^{3}$
}

\author{
${ }^{1}$ Department of Obstetrics \& Gynecology, All India Institute of Medical Sciences, Bhubaneswar-757019, Orissa, India \\ ${ }^{2}$ Department of Pathology and Laboratory Medicine, All India Institute of Medical Sciences Bhubaneswar-757019, \\ Orissa, India \\ ${ }^{3}$ Department of Radiodiagnosis, All India Institute of Medical Sciences Bhubaneswar-757019, Orissa, India
}

Received: 30 March 2015

Accepted: 05 May 2015

\section{*Correspondence: \\ Dr. Kameshwarachari Pushpalatha, \\ E-mail: pushpak_73@yahoo.com}

Copyright: (c) the author(s), publisher and licensee Medip Academy. This is an open-access article distributed under the terms of the Creative Commons Attribution Non-Commercial License, which permits unrestricted non-commercial use, distribution, and reproduction in any medium, provided the original work is properly cited.

\begin{abstract}
Mesonephric cyst is a vestigial remnant of the Wolffian duct in females. Occasionally, the mesonephric duct persists lateral to the vagina and uterus, and cystic lesions may sometimes develop into neoplastic lesions. Mesonephric cysts are very rare. Here we report an unprecedented large left broad ligament mesonephric cyst in a 27 year old lady that posed diagnostic and surgical challenges. Ultrasound findings included cystic swelling probably arising from left ovary suggestive of left ovarian cyst. Plain abdominal computed tomography scan showed large simple left paraovarian cystic mass with no solid areas. During laparotomy, we found a large left thin walled cyst with smooth external surface with vascular prominence measuring $20 \times 13 \times 11 \mathrm{~cm}$ arising within the broad ligament stretching the left Fallopian tube, close to left ovary and adherent to recto-sigmoid. Cystectomy was done with preservation of left ovary. Histopathology report was consistent with mesonephric cyst. Mesonephric duct cyst should be considered in the diagnosis of abdomino-pelvic masses in females of adolescent and early reproductive age.
\end{abstract}

Keywords: Large Mesonephric cyst, Surgical excision, Ovarian preservation, Renal anomalies

\section{INTRODUCTION}

Mesonephric cyst is a vestigial remnant of the Wolffian duct in females. However, if the cellular lining remains active, it may lead to cystic lesions that may cause pain or torsion of the adnexa. Occasionally, the mesonephric duct persists lateral to the vagina and uterus, and cystic lesions may sometimes develop into neoplastic lesions. Mesonephric cysts are very rare. ${ }^{1}$

Here we report an unprecedented large left broad ligament mesonephric cyst in a 27 year old lady that posed diagnostic and surgical challenges.

\section{CASE REPORT}

A 27 year old female, graduate, married for 6 months, presented to our gynaecologic clinic with complaints of large abdominal mass of 5 years duration. She initially felt the mass in the lower abdomen which gradually increased in size over the time to surpass the umbilicus. Patient had no bladder and bowel symptoms, cough or pain abdomen. Her previous menstrual cycles were regular and normal with respect to cycle length, duration and amount of flow. Her past history was nothing significant. On general physical examination, patient was moderately built and nourished. Systemic examination with regard to respiratory system, cardio-vascular system, breast, bone and thyroid were normal. Abdominal examination revealed a huge abdomino-pelvic cystic mass of $28 \times 20 \mathrm{~cm}$ with regular margins and smooth 
surface. Vulval and per-speculum examination showed normal features. On bimanual examination, cystic mass was felt through the anterior, bilateral fornices. Uterus felt normal in size and position. There was no tenderness in any of the fornices. Per rectal examination showed normal Pouch of Douglas and smooth rectal mucosa.

\section{Whole abdomen ultrasound (Transabdominal followed by transvaginal ultrasound)}

Uterus measured $8.4 \times 4.6$ × $3 \mathrm{~cm}$ with endometrial thickness of $3.8 \mathrm{~mm}$. Right ovary measured $2 \times 1.6 \mathrm{~cm}$. Left ovary was not separately visible but large simple left sided cystic mass reaching above the umbilicus was noted. Upper abdominal organs including bilateral kideneys were normal. No ascites. No enlarged retroperitoneal lymphadenopathy.

\section{Plain abdominal CT scan}

Liver, spleen, gallbladder, adrenal glands, kidneys, and pancreas were normal. A large simple left paraovarian cystic mass with no solid areas. Bilateral ovaries were seen separately with normal size. Uterus measured $8.4 \mathrm{x}$ $4.6 \times 3 \mathrm{~cm}$ with endometrial thickness of $3.8 \mathrm{~mm}$.

Surgery was proposed to the patient in view of the large abdominal mass of paraovarian origin. Consent was taken for laparotomy and cystectomy. Laparotomy was performed with midline incision.

\section{Intraoperative findings}

No ascites with normal omentum, intestines and upper abdominal organs. A large left cystic mass was seen arising within the broad ligament stretching the left Fallopian tube, close to left ovary was found. Mass was adherent to Recto-Sigmoid. Uterus and right side tube and ovary were normal. Cyst was separated from the recto-sigmoid and left ovary using sharp dissection. The cyst was excised along with the left Fallopian tube by clamping at the large pedicle. Left ovary was preserved.

\section{Pathology}

\section{Gross description (Figure 1)}

A thin walled cyst with smooth external surface with

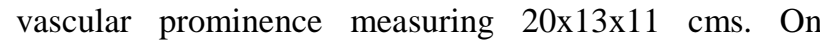
cutting open the unilocular cyst, serous fluid oozed out. Intracystic wall was smooth without any projections or solid areas.

\section{Microscopy (Figure 2)}

A cyst lined by single layer of flattened to cuboidal epithelium with smooth muscle in the wall. Focal area shows stratification. There was no atypia or malignancy in the section studied. The final histopathological features were consistent with mesonephric cyst.

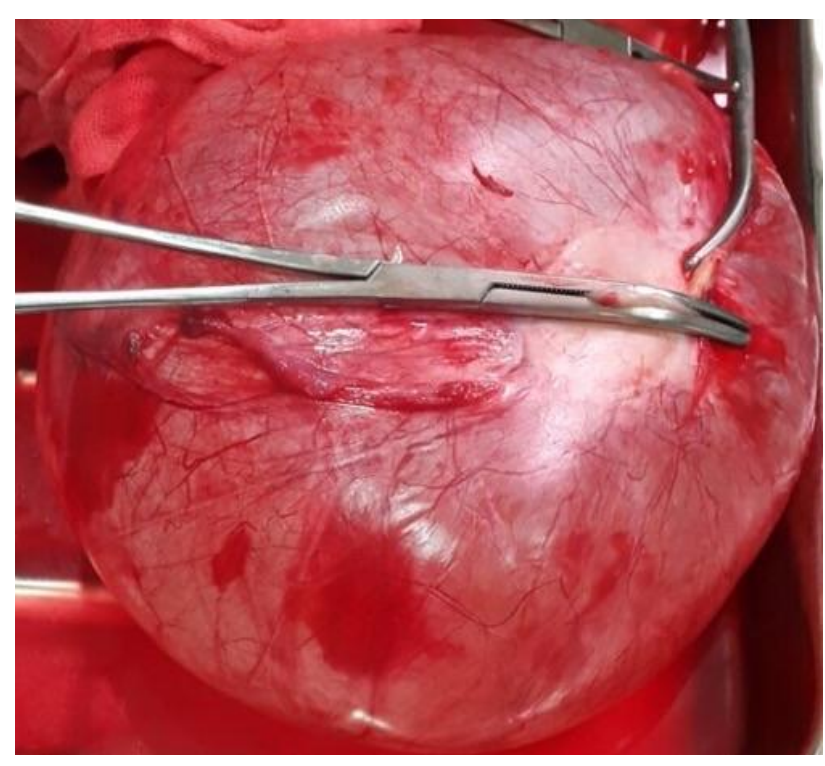

Figure 1: Macroscopic features of mesonephric cyst.

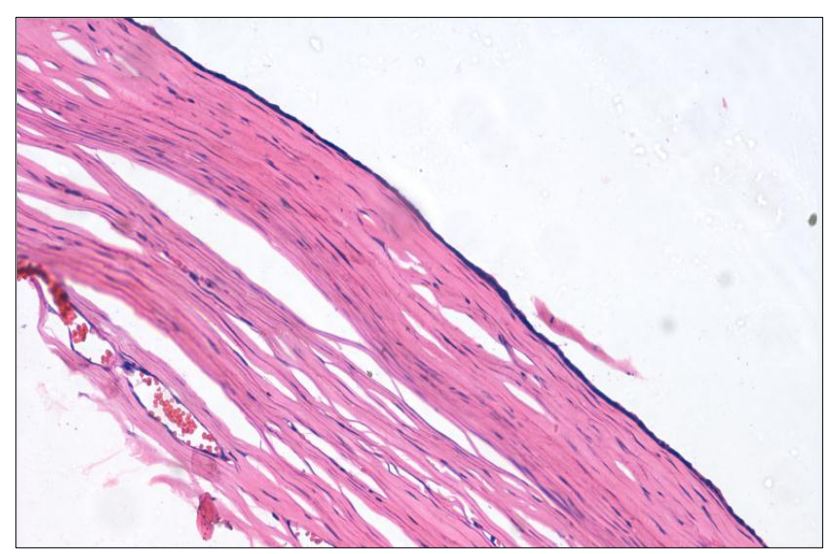

Figure 2: Microscopic features showing cyst lined by single layer of flattened to cuboidal epithelium with smooth muscle in the wall.

\section{DISCUSSION}

In females, mesonephric ducts remain as vestigial structures and are located in the broad ligaments but occasionally persist lateral to the vagina and uterus, and may develop into cystic or neoplastic lesions causing pain and torsion. There are fewer than 100 cases reported in the literature, largest measuring $10 \times 8.5 \times 3.2 \mathrm{~cm} .{ }^{1}$ Some are associated with an absent kidney on the ipsilateral side of the cyst necessitating evaluation of the renal system when this entity is diagnosed. ${ }^{2,3}$ Though malignant change is very rare, Kyung et al. has reported a case of adenocarcinoma arising in a vaginal Müllerian cyst in a 48 year old woman. So the possibility of malignant transformation also should be considered and careful follow-up is warranted. ${ }^{4,5}$ The present case is unique owing to unprecedented size coupled with diagnostic and surgical challenges. However left ovary was completely preserved for promising obstetric prospects. 
Funding: No funding sources

Conflict of interest: None declared

Ethical approval: Not required

\section{REFERENCES}

1. Akkawi R, Valente AL, Badawy SZ. Large mesonephric cyst with acute adnexal torsion in a teenage girl. J Pediatr Adolesc Gynecol. 2012 Dec;25(6):e143-5.

2. Gadbois WF, Duckett JW Jr. Gartner's duct cyst and ipsilateral renal agenesis. Urology. 1974;4:720.
3. Hardeman DR, De Groat A. Cyst of Wolffian origin with aplasia of kidney and ureter, hydronephrosis, and undescended testicle. Ann Surg. 1936;103:388.

4. Lee KS, Park KH, Lee S, Kim JY, Seo SS. Adenocarcinoma arising in a vaginal Müllerian cyst: a case report. Gynaecol Oncol. 2005;99:767-9.

5. Samal S, Mahapatro A, Poorkodi B. Posterior vaginal wall cyst of Mullerian origin: a case report. Int J Reprod Contracept Obstet Gynecol. 2015;4:245-6.

DOI: $10.18203 / 2320-1770$. ijrog 20150125

Cite this article as: Pushpalatha K, Mishra P, Bag ND.

An unprecedented large mesonephric cyst posing diagnostic dilemma and surgical challenge. Int $\mathbf{J}$ Reprod Contracept Obstet Gynecol 2015;4:905-7. 